\title{
Molecular Docking Investigation of Antiviral Herbal Compounds as Potential Inhibitors of SARS-CoV-2 Spike Receptor
}

\author{
Mahdi Soleyman Fallah ${ }^{1}$ (D), Mohammad Bayati ${ }^{1}{ }^{(D)}$, Ali Najafi ${ }^{2}$ (D) , Esmael Behmard ${ }^{2}$ (D), Seyed Javad \\ Davarpanah $^{1, *}$ (D) \\ 1 Applied Biotechnology Research Center, Baqiyatallah University of Medical Sciences, Tehran, Iran \\ 2 Molecular Biology Research Center, Systems Biology and Poisonings Institute, Baqiyatallah University of Medical \\ Sciences, Tehran, Iran \\ * Correspondence: davarpanah@bmsu.ac.ir;
}

Scopus Author ID 57191964787

Received: 28.12.2020; Revised: 21.01.2021; Accepted: 24.01.2021; Published: 31.01.2021

\begin{abstract}
At the beginning of 2020, a new type of Coronavirus (Severe Acute Respiratory Syndrome Coronavirus -2 (SARS-CoV-2)) dismayed the world and led to public health emergencies. This virus has caused a remarkable percentage of morbidity and mortality. Also, the lack of an effective treatment to fight this virus is another concern that should be given attention. Herbal medicines and purified natural products have been reported for their antiviral activity against SARS-CoV-2. In this study, molecular docking of effective compounds in the extracts and essential oils of Zingiber officinale, Glycyrrhiza glabra Sambucus nigra, Panax ginseng Ocimum basilicum, and Origanum vulgare was carried out to investigate their binding to the X-ray structure of the ACE2 binding domain of SARSCoV-2. The Glide docking program was utilized for molecular docking with standard precision (SP) and extra precision (XP). Finally, 7 compounds- mainly belong to Panax ginseng-showed a higher docking score than some known antiviral compounds. Floralginsenoside B, which is extracted from Panax ginseng, indicated a strong binding affinity $(-8.618 \mathrm{kcal} / \mathrm{mol})$ to the crucial residues of the receptor binding domain of SARS-CoV-2 comparing to Doravirine $(-7.2 \mathrm{kcal} / \mathrm{mol})$, Hetacillin $(-7.1$ $\mathrm{kcal} / \mathrm{mol})$, Ketoprofen $(-7.0 \mathrm{kcal} / \mathrm{mol})$, and Mefloquine $(-7.0 \mathrm{kcal} / \mathrm{mol})$ reported in previous articles. Based on the excellent binding affinities of these herbal compounds, we concluded that these phytochemicals could be promising candidates for fighting against the COVID-19 pandemic.
\end{abstract}

Keywords: herbal compounds; molecular docking; SARS- CoV-2; COVID-19; binding affinity.

(C) 2021 by the authors. This article is an open-access article distributed under the terms and conditions of the Creative Commons Attribution (CC BY) license (https://creativecommons.org/licenses/by/4.0/).

\section{Introduction}

In December 2019, for the first time in Wuhan, China, a new type of coronavirus was identified after being infected people with pneumonia for no apparent reason while existing vaccines and treatments were ineffective [1]. According to the World Health Organization, the new SARS- CoV (SARS- CoV-2) has killed more than 1,740,390 people. About 78,383,527 people have been infected with the virus in 220 countries by December 26 (2020), indicating the high spread of the epidemic corona worldwide. Symptoms including fever, dry cough, and sometimes respiratory problems such as asthma, dyspnea, sore throat, and runny nose [2]. SARS-CoV-2 is a coated virus with a genome of ribonucleic acid and a length of $29.8 \mathrm{k}$ base. The virus genome contains 14 open reading frames (ORF) that encode 27 proteins. The 
ORF1ab and ORF1a genes at the end of the five genomes encode the pp1ab and pp1a proteins, respectively. They express 15 non-structural proteins nsp1-nsp10 and nsp12-nsp16.

On the other hand, four structural proteins, including spike surface protein $(S)$, an envelope protein $(\mathrm{E})$, membrane protein $(\mathrm{M})$, nucleocapsid protein $(\mathrm{N})$, and eight sub-proteins including 3a, 3b, p6,7a, 7b, 8b, 9b and ORF14 are encoded at the end of 3 genomes [3, 4]. One of the known mechanisms for SARS virus entry into the cell is binding a unique protein at the surface of the virus membrane to a receptor located at the host cell's surface. This protein, called Spike, binds to the enzyme "ACE2", which enters the host cell [5, 6]. Accordingly, reported studies show that the COVID-19 has a similar mechanism for entering the host cell through the ACE2 receptor.

There are many herbs with excellent antiviral effects. Nowadays, medicinal plants and herbal compounds have attracted much attention due to their easy access and low side effects compared to synthetic drugs. Researches show that plant extracts have interesting antiviral properties. Moreover, the antiviral activity of flavonoids and anthraquinones has been investigated [7-11]. It has been reported that the Hypericin and pseudohypericin obtained from the plant species Hypericum has significant antiviral properties [12]. Anthraquinones with polyphenolic or polysulfone substitution inhibit the HIV-1 reverse transcriptase enzyme [13].

The docking process leads to an understanding of the relationship of structure-activity of known ligands, based on the level of molecular interaction with the receptor's active site [14, 15]. One of the molecular docking applications is designing drugs based on structure. Docking has minimized the required time to identify and design medicinal compounds, their type, and optimize their structure [16].

Investigation of plant compounds' effect on the SARS-CoV-2 life cycle using docking methods has been reported in many studies. In 2020, Dev Sharma et al. reported that 1,8 cineole extracted from Eucalyptus camaldulensis inhibit the function of the SARS-CoV-2 protease enzyme by binding energy $-6.4 \mathrm{kcal} / \mathrm{mol}$ [17]. Research by Talia et al. using molecular docking has shown that the compounds histidine, folic acid, and lepidine can disrupt the life cycle of the SARS-CoV-2 by inhibiting the protease enzyme. These natural compounds act as drug candidates [18]. Also, Hendra et al. investigated, using docking software, the effect compounds of several herbal plants on the SARS-CoV-2 protease enzyme. Finally, it was found that the compounds kaempferol, quercetin, naringenin, curcumin have a more negative binding energy than other compounds, so they can inhibit the enzyme protease of SARS-CoV-2. However, this hypothesis requires further experiments in the biological environment of the human body [19].

According to a 2020 study, some of the drugs included Ribavirin, Remdesivir, Sofosbuvir, Galidesivir, and Tenofovir, which were available on the market or in clinical trials identified as potential antiviral drugs. Besides, the compounds IDX-184, Sofosbuvir, and Yak showed excellent results against SARS-CoV-2 [20]. In the research of A. Belhassan et al., from 67 different selected compounds, the active substances of Crocus sativus, Nerium oleander, and Foeniculum vulgare plants included: crocin, digitoxigenin, and beta-eudesmol, interact with the active site of the target receptor (the main protease of SARS-CoV-2) by the binding energy amount of $-8.2,-7.2$ and $-7.1 \mathrm{kcal} / \mathrm{mol}$ respectively [21]. Examining existing drugs is the only possible option for responding quickly to the global challenge of Covid 19. By examining the effect of 48 effective SARS-CoV drugs on the SARS-CoV-2, Lee et al. identified the 24 drug candidates with antiviral ability against SARS-CoV-2 infections. Some of these drug candidates have inhibited less than $50 \%$. Two drugs called niclosamide and ciclesonide approved by the World Health Organization particularly attract much attention [22]. This 
research aims to investigate the molecular docking of effective compounds in the extracts and essential oils of some herbs (Zingiber officinale, Glycyrrhiza glabra, Sambucus nigra, Panax ginseng, Ocimum basilicum, and Origanum vulgare) to the ACE2 binding domain of SARSCoV-2 spikes and to compare their interactions in terms of energy with known antiviral compounds such as Doravirine, Hetacillin, Ketoprofen, and Mefloquine. Sruthi Unni et al. previously Observed that these compounds, which had been used as other treatments (Doravirine as an inhibitor of the HIV genome [23], Hetacillin as an antibiotic [24], Ketoprofen as an analgesic [25], and Mefloquine as an anti-malarial agent [26]) exhibited Coronavirus inhibition through computer calculations. They have shown these compounds could successfully inhibit the virus spike proteins. In the present work, we seek to study this type of interaction with the mentioned herbal compounds.

\section{Materials and Methods}

\subsection{Protein preparation.}

SARS-CoV-2 spike receptor-binding domain (RBD) crystal structure (PDBID: 6M0J) was obtained from the protein data bank. The protein preparation wizard module was applied to prepare the crystal structure; missing loops and side chains were fixed using Prime, adding hydrogens to amino acids, creating disulfide bonds between sulfur atoms, and deleting water molecules within $3.00 \AA$ distance of HET groups were performed for this purpose. Undetermined groups such as hydroxyl and amides in Asn and Glu amino acids need to orient by optimization. The PROPKA was used in $\mathrm{pH} 7.00$ to optimize protonation states of amino acid residues. Eventually, the spike structure was minimized by an OPLS3 force field.

\subsection{Ligand preparation.}

The structure of natural products present in essential oil and extraction of desired plants was downloaded from PubChem (https://pubchem.ncbi.nlm.nih.gov) and Spectra Base (https://spectrabase.com/compound). More than 320 compounds were prepared using the LigPrep module of Maestro 10.2 suite 2015. The initial 2D compounds were changed to 3D ones. At most 5 conformers of each compound were created, as well as the molecules were desalted. At the end of the process, entire compounds were minimized utilizing the OPLS3 force field.

\subsection{Molecular docking.}

Molecular docking of entire compounds was carried out with Glide of Maestro 10.2 in flexible ligand mode. At first, the prepared ligands were docked using SP (standard precision) option. Redocking $10 \%$ of hit poses was performed using XP (extra precision) as a more accurate docking procedure. Eventually, the results of molecular docking are expressed as $\mathrm{Kcal} / \mathrm{mol}$.

\subsection{Grid generation.}

The active binding site of the SARS-CoV-2 spike structure was formed around amino acids including Ile 472, Lys458, Asp467, Gly474, Arg457, and Ser459 using Glide of Maestro suite 2015. First of all, the ACE2 bounded to the SARS-CoV-2 Spike was removed, then the grid box was created in the binding position. Validating of active site was evaluated at 
coordinates of (X: -38.48, Y: 42.50, Z: 13.48), and grid spacing of (X, Y, Z: $20 \AA$ ) utilizing site map tool.

\section{Results and Discussion}

In this study, the inhibitory effect of compounds in the extracts and essential oils of Zingiber officinale, Glycyrrhiza glabra, Sambucus nigra, Panax ginseng, Ocimum basilicum, and Origanum vulgare against the SARS-CoV-2 Spike RBD crystal structure (PDBID: 6M0J) was investigated using molecular modeling. Extra precision (XP) of the Schrödinger maestro suite (version 11.8, 2018) was utilized for molecular docking validation. The order of strength of binding was as follows: Floralginsenoside $\mathrm{B}>$ Floralginsenoside $\mathrm{E}>\mathrm{Cyanidin}-3$-rutinoside $>$ Floralquinquenoside $\mathrm{D}>$ Floralginsenoside $\mathrm{C}>$ Floralginsenoside $\mathrm{F}>$ Galangin-3-rutinoside.

The top 7 ligand molecules with their docking score and different interactions with amino acids of the SARS-CoV-2 spike RBD pocket are listed in Table 1. Interactions between the active compounds and the receptor-binding domain were shown in Figures 1-7.

In 2020, Sruthi Unni et al. worked on the binding affinity of some approved drugs against Spike protein of human SARS-CoV-2 and reported Doravirine, Hetacillin, Ketoprofen, and Mefloquine as hit molecules due to their good binding affinity to the active site of the Spike binding domain [27]. In Table 1, as shown, Floralginsenoside B, Floralginsenoside E, Cyanidin-3-rutinoside, Floralquinquenoside D, and Floralginsenoside $\mathrm{C}$ with docking score > -7.3 showed high binding affinity more than hit molecules reported by Sruthi Unni et al.

Table 1. Glide docking energy of top seven compounds against the COVID-19 receptor-binding domain.

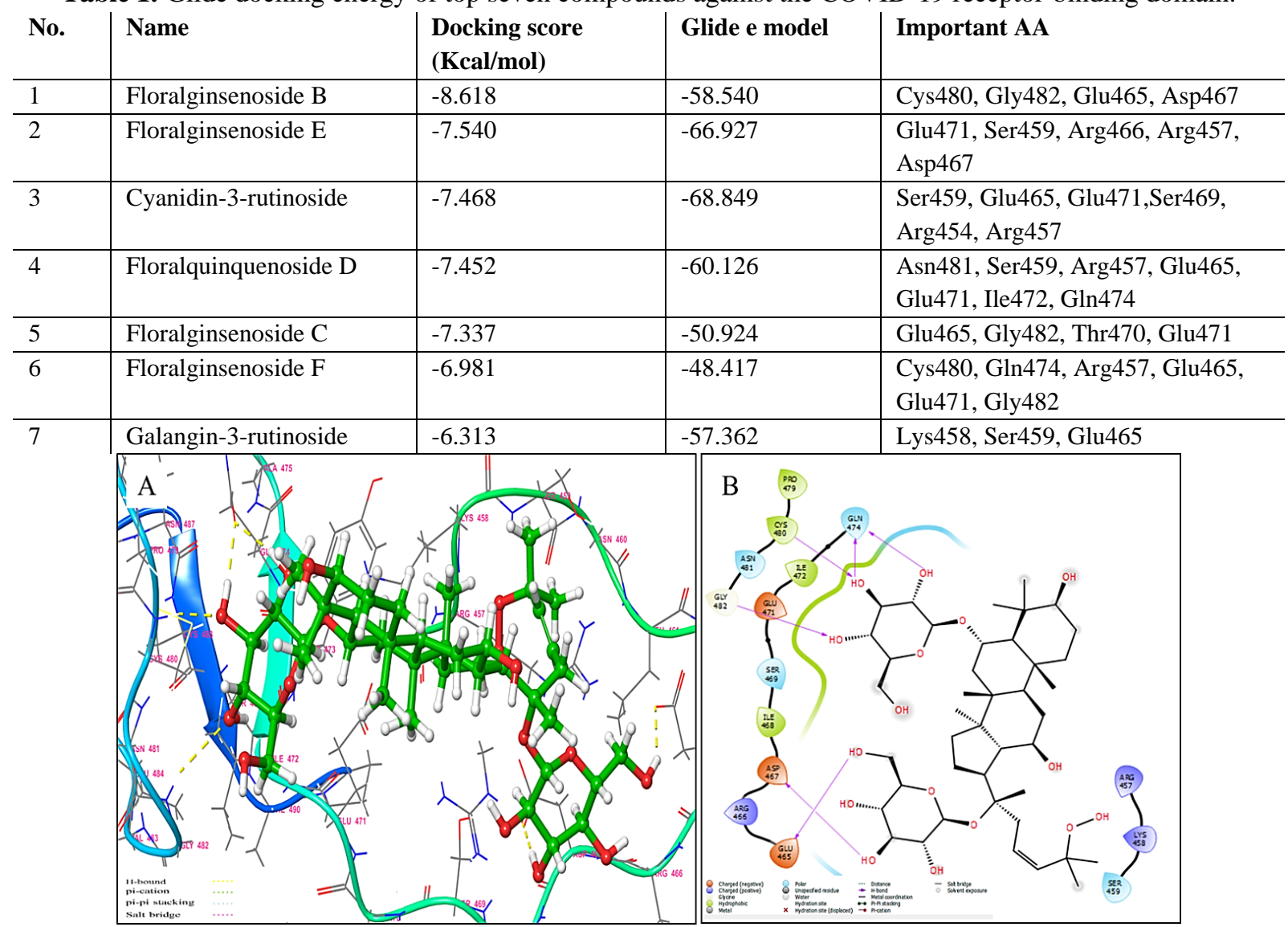

Figure 1. The binding pose of the receptor-binding domain - Floralginsenoside B complex. (A) 3D interaction; (B) 2D interaction. The 2D interaction map indicates the importance of the involved amino acid in the binding pocket. 
Floralginsenoside B (essential oils of Panax ginseng) with a docking score of -8.61 $\mathrm{Kcal} / \mathrm{mole}$ indicated high binding affinity than other compounds against the SARS-CoV-2 Spike RBD. Docking analysis showed that this compound interacts with active pocket residues of 6M0J mainly through hydrogen bonds with Gln474, Cys 480, Gly 482, Glu465, and Asp467 (Figure 1).

Floralginsenoside E (essential oils of Panax ginseng) showed a binding score of -7.54 $\mathrm{Kcal} / \mathrm{mole}$ with the formation of six H-bonds with Glu471, Ser 459, Arg466, Arg 457, and Asp467 (Figure 2).

Docking results of Cyanidin-3-rutinoside (essential oils of Sambucus nigra) depict that this compound with the docking score $-7.46 \mathrm{Kcal} /$ mole interacts with the binding pocket $6 \mathrm{M} 0 \mathrm{~J}$ through hydrogen bonds as well as $\pi-\pi$ and $\pi$-cation interactions. Amino acid residues Ser459, Glu465, Glu471, and Ser469 establish hydrogen bound with this compound, and amino acid residues Arg 454, as well as Arg 457, establish p-p and p-cation interactions (Figure 3).

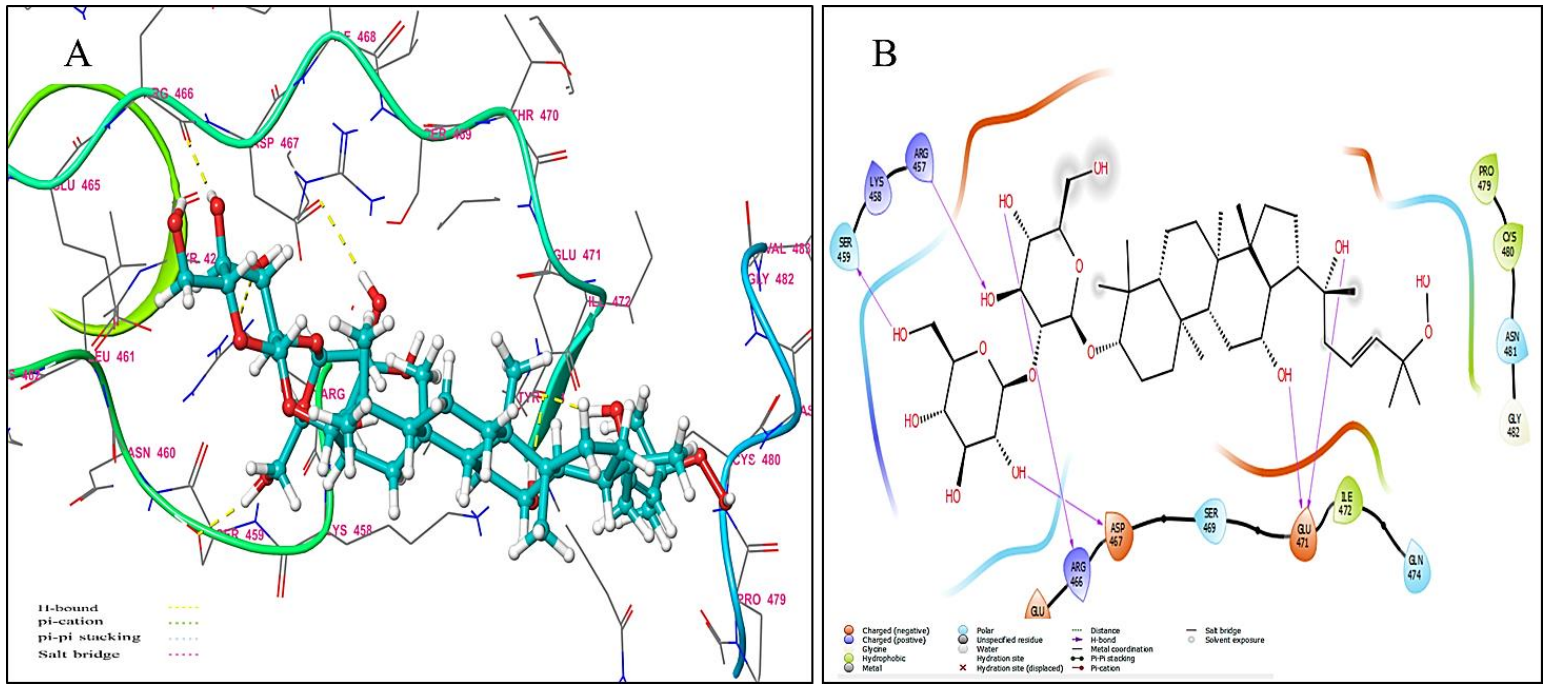

Figure 2. The binding pose of the receptor-binding domain - Floralginsenoside E complex. (A) 3D interaction; (B) 2D interaction. The 2D interaction map indicates the importance of the involved amino acid in the binding pocket.

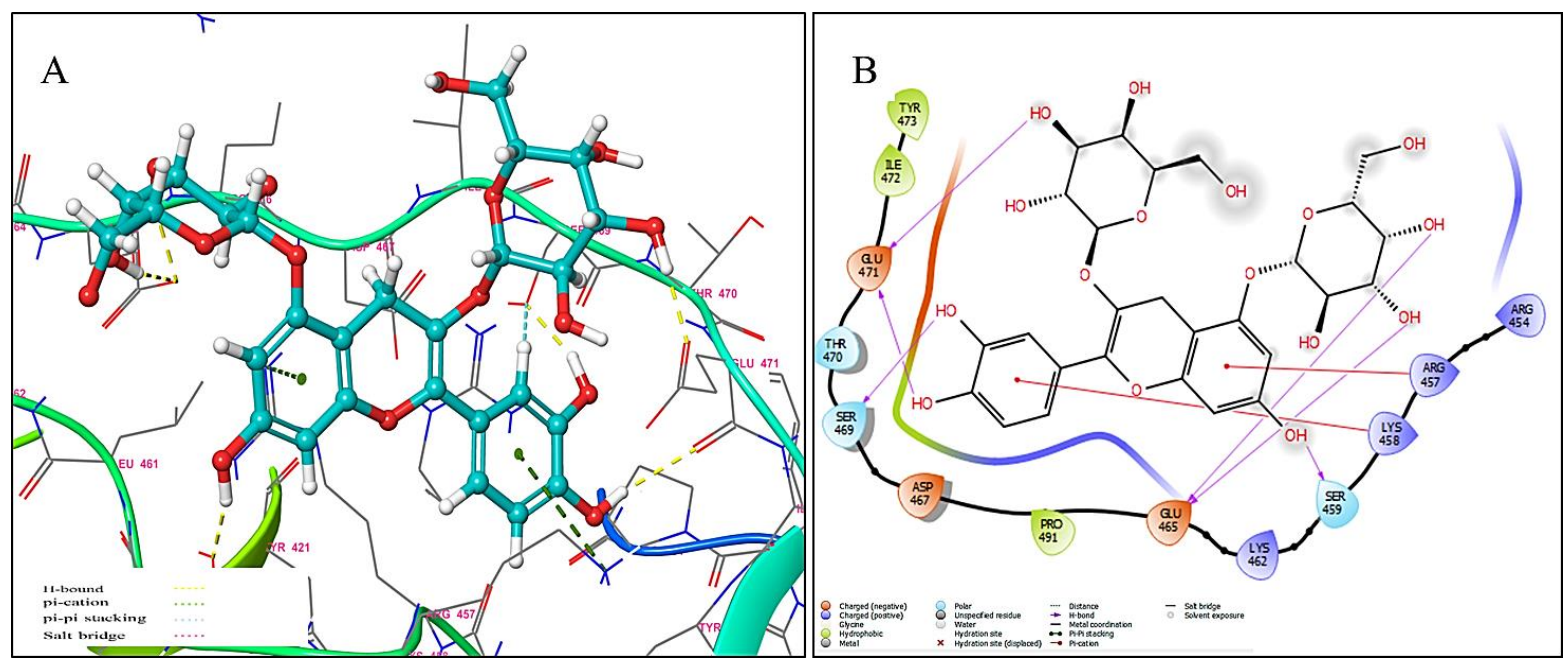

Figure 3. The binding pose of the receptor-binding domain - Cyanidin-3-rutinoside complex. (A) 3D interaction; (B) 2D interaction. The2D interaction map indicates the important involved amino acid in the binding pocket.

Floralquinquenoside D (essential oils of Panax ginseng) indicated the docking score $7.45 \mathrm{Kcal} / \mathrm{mole}$. This compound interacts with active site residues of $6 \mathrm{M} 0 \mathrm{~J}$ through 9 hydrogen 
bonds with amino acid residues Asn 481, Ser 459, Arg 457, Glu 465, Glu 471, Ile 472, and Gln 474 (Figure 4).

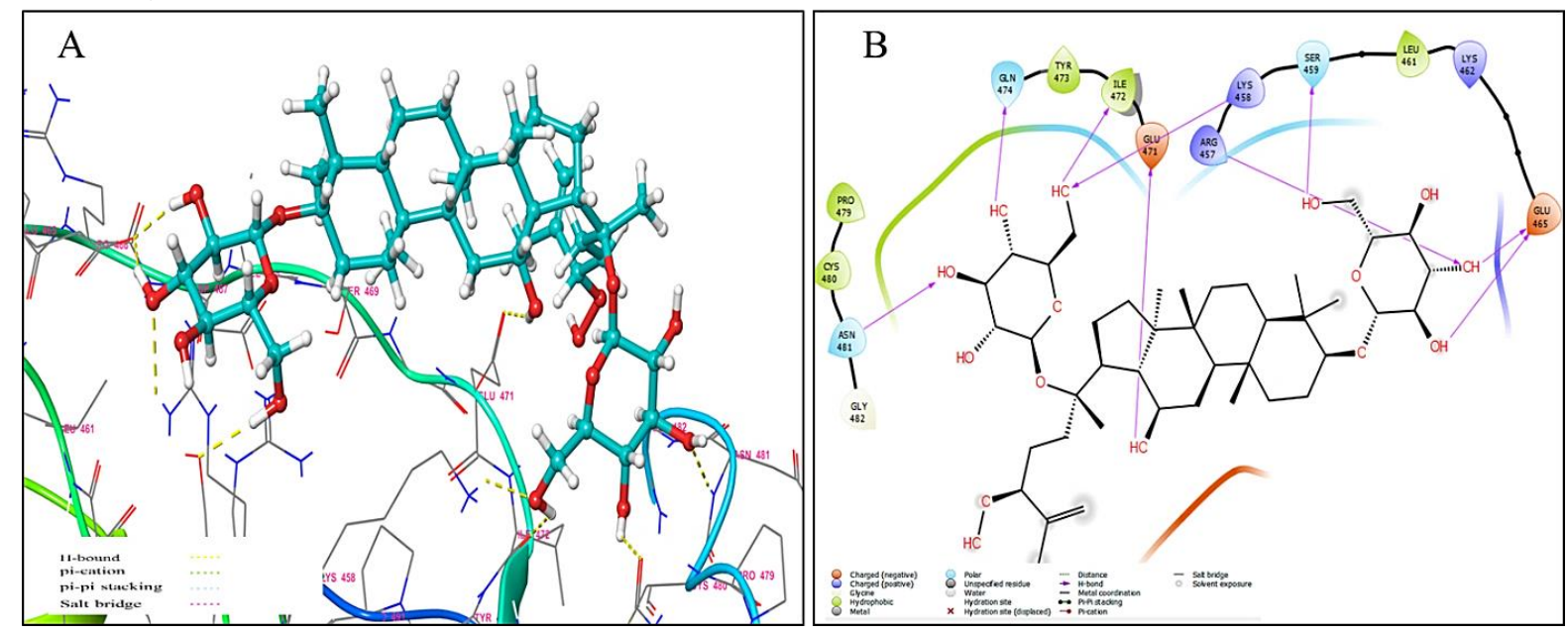

Figure 4. The binding pose of the receptor-binding domain - Floralquinquenoside D complex. (A) 3D interaction; (B) 2D interaction. The2D interaction map indicates the important involved amino acid in the binding pocket.

The analysis of docking interactions of Floralginsenoside $\mathrm{C}$ (essential oils of Panax ginseng) with binding score $-7.33 \mathrm{Kcal} /$ mole revealed that the major interactions involve hydrogen bonds with amino acid residues Glu 465, Gly 482, Thr 470, and Glu 471 (Figure 5).
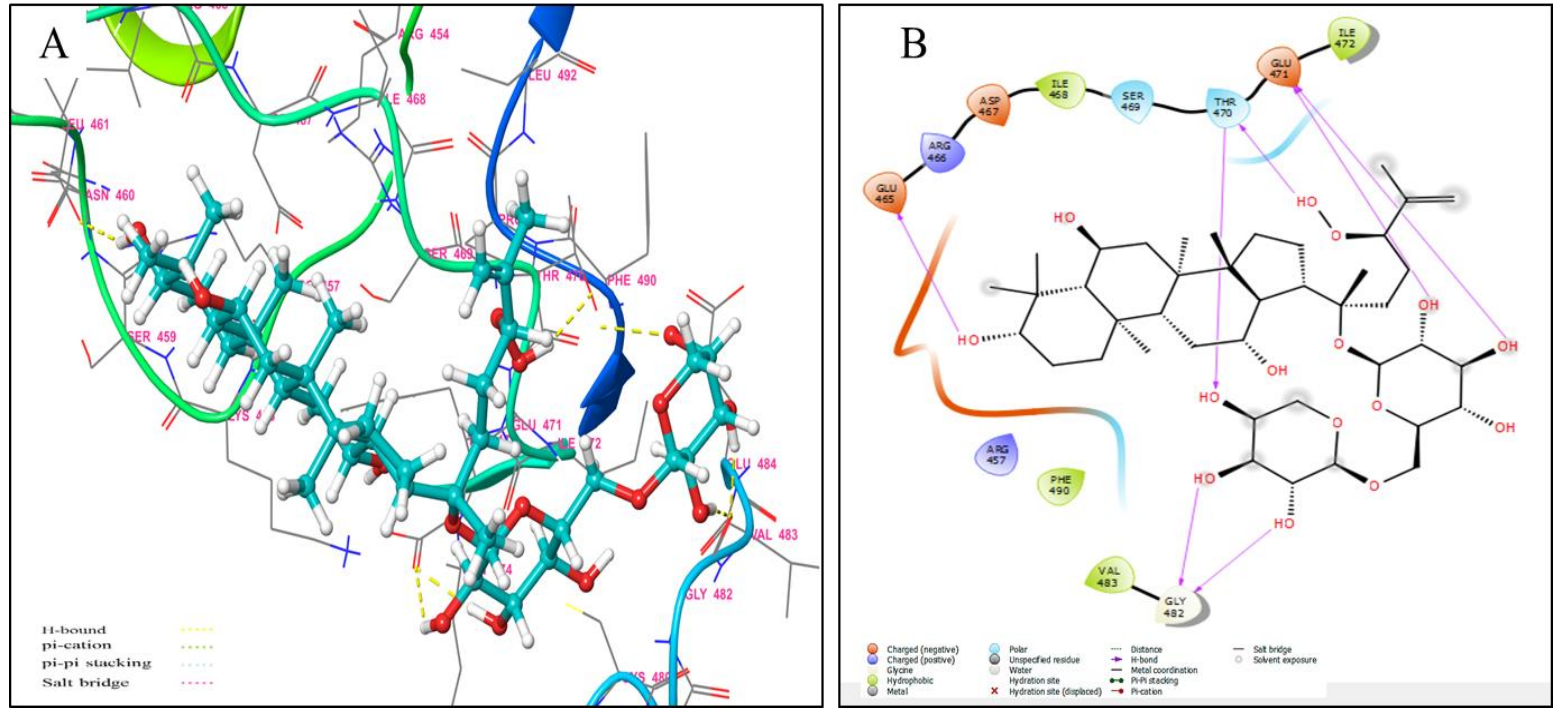

Figure 5. The binding pose of the receptor-binding domain - Floralginsenoside C complex. (A) 3D interaction; (B) 2D interaction. The2D interaction map indicates the important involved amino acid in the binding pocket.

Figure 6 depicts Floralginsenoside F's interactions (essential oils of Panax ginseng) with the binding pocket of $6 \mathrm{M} 0 \mathrm{~J}$. Summarizing the interactions between the amino acid residues of 6M0J and Floralginsenoside F, Cys 480, Gln 474, Arg 457, Glu 465, Glu 471, and Gly 482 were found to be the most frequent hydrogen bounds.

Galangin-3-rutinoside with docking score $-6.31 \mathrm{Kcal} / \mathrm{mole}$ interact with active site residues of 6M0J mainly through hydrogen bonds. Amino acid residues Glu 465, Ser 459, and Lys458 play a major role in docking interaction (Figure 7). 

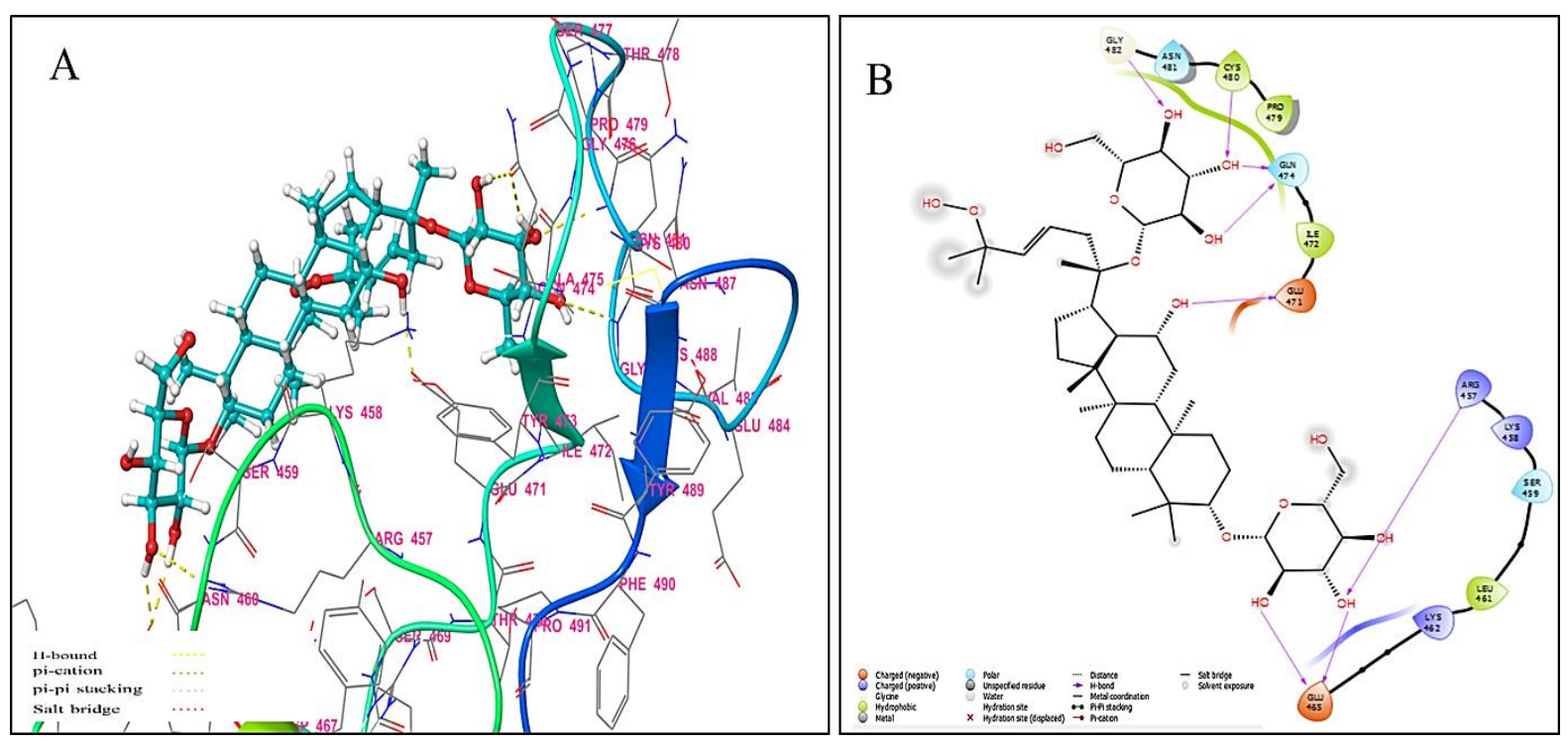

Figure 6. The binding pose of the receptor-binding domain - Floralginsenoside F complex. (A) 3D interaction; (B) 2D interaction. The2D interaction map indicates the important involved amino acid in the binding pocket.
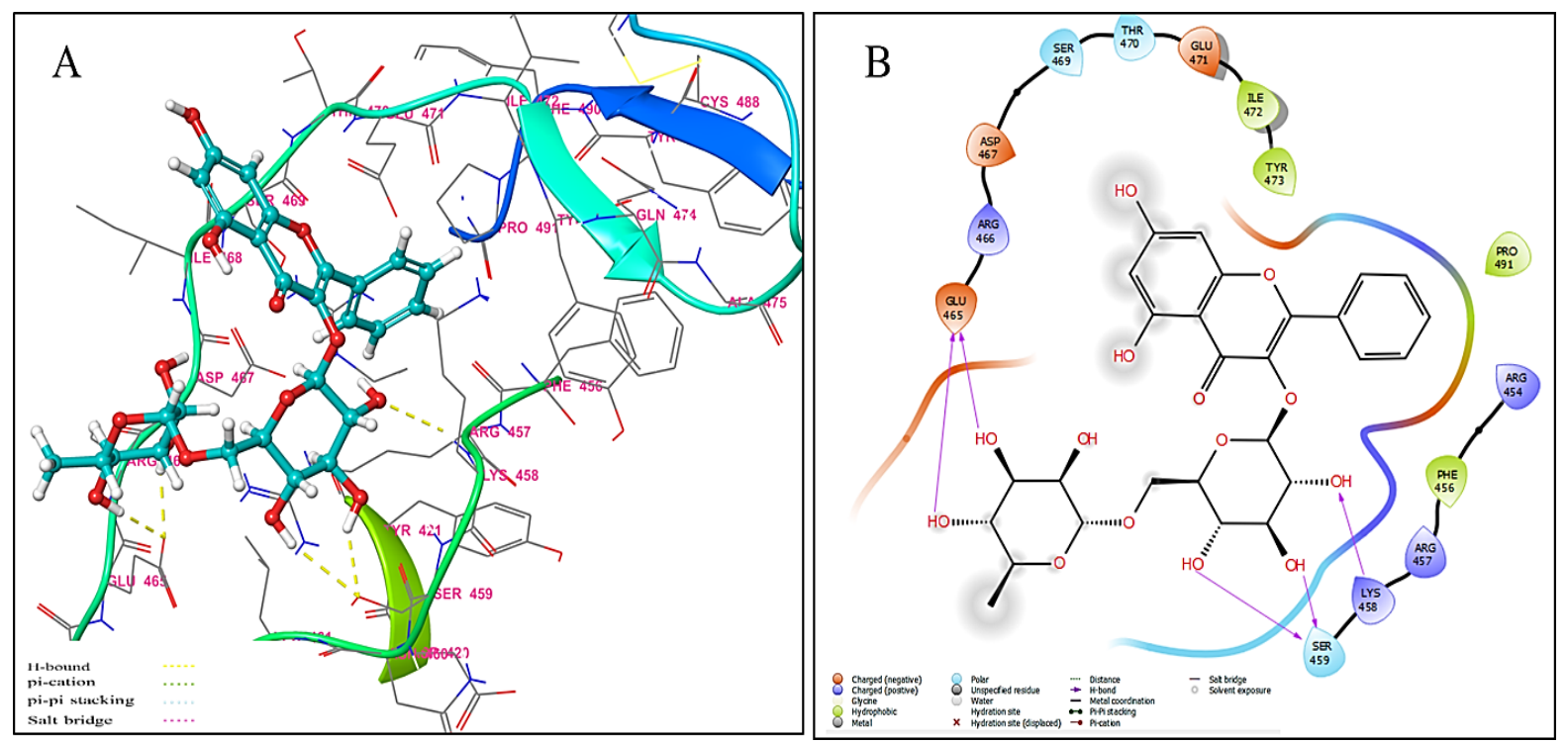

Figure 7. The binding pose of the receptor-binding domain - Galangin-3-rutinoside complex. (A) 3D interaction; (B) 2D interaction. The2D interaction map indicates the important involved amino acid in the binding pocket.

\section{Conclusions}

The X-ray structure of the ACE2 binding domain of SARS-CoV-2 and effective compounds in the extracts and essential oils of Zingiber officinale, Glycyrrhiza glabra, Sambucus nigra, Panax ginseng, Ocimum basilicum, and Origanum vulgare were utilized for docking studies to retrieve natural lead compounds. Finally, 7 ligand molecules- belong to the extracts and essential oils of Panax ginseng and Sambucus nigra-showed good binding affinity against the SARS-CoV-2 spike RBD pocket and were chosen as hit compounds based on the consensus scoring function. From the overall analyses, we can conclude that the extracts and essential oils of Panax ginseng could be introduced as promising COVID-19 inhibitors.

\section{Funding}

This research received no external funding. 


\section{Acknowledgments}

The authors gratefully acknowledge support of this work by the Applied Biotechnology Research Center and Molecular Biology Research Center, Baqiyatallah University of Medical Sciences, Tehran, Iran.

\section{Conflicts of Interest}

The authors declare no conflict of interest.

\section{References}

1. Casanova, L.M.; Jeon, S.; Rutala, W.A.; Weber, D.J.; Sobsey, M.D. Effects of air temperature and relative humidity on coronavirus survival on surfaces. Appl. Environ. Microbiol. 2010, 76, 2712-2717, https://doi.org/10.1128/AEM.02291-09.

2. Ganyani, T.; Kremer, C.; Chen, D.; Torneri, A.; Faes, C.; Wallinga, J.; Hens, N. Estimating the generation interval for coronavirus disease (COVID-19) based on symptom onset data, March 2020. Eurosurveillance 2020, 25, 2000257, https://doi.org/10.2807/1560-7917.es.2020.25.17.2000257.

3. Wu, A.; Peng, Y.; Huang, B.; Ding, X.; Wang, X.; Niu, P.; Meng, J.; Zhu, Z.; Zhang, Z.; Wang, J. Genome composition and divergence of the novel coronavirus (2019-nCoV) originating in China. Cell Host Microbe 2020, 27, 325-328, https://doi.org/10.1016/j.chom.2020.02.001.

4. Akbariqomi, M.; Hosseini, M.S.; Rashidiani, J.; Sedighian, H.; Biganeh, H.; Heidari, R.; Moghaddam, M.M.; Farnoosh, G.; Kooshki, H. Clinical characteristics and outcome of hospitalized COVID-19 patients with diabetes: A single-center, retrospective study in Iran. Diabetes Res. Clin. Pract. 2020, 169, 108467, https://doi.org/10.1016/j.diabres.2020.108467.

5. Li, W.; Moore, M.J.; Vasilieva, N.; Sui, J.; Wong, S.K.; Berne, M.A.; Somasundaran, M.; Sullivan, J.L.; Luzuriaga, K.; Greenough, T.C. Angiotensin-converting enzyme 2 is a functional receptor for the SARS coronavirus. Nature 2003, 426, 450-454.

6. Heiat, M.; Hashemi-Aghdam, M.-R.; Heiat, F.; Rastegar Shariat Panahi, M.; Aghamollaei, H.; Moosazadeh Moghaddam, M.; Sathyapalan, T.; Ranjbar, R.; Sahebkar, A. Integrative role of traditional and modern technologies to combat COVID-19. Expert Rev. Anti Infect. Ther. 2020, 1-11, https://doi.org/10.1080/14787210.2020.1799784.

7. Andersen, D.O.; Weber, N.D.; Wood, S.G.; Hughes, B.G.; Murray, B.K.; North, J.A. In vitro virucidal activity of selected anthraquinones and anthraquinone derivatives. Antiviral Res. 1991, 16, 185-196, https://doi.org/10.1016/0166-3542(91)90024-L.

8. Hayashi, K.; Hayashi, T.; Morita, N. Mechanism of action of the antiherpesvirus biflavone ginkgetin. Antimicrob. Agents Chemother. 1992, 36, 1890, https://doi.org/10.1128/AAC.36.9.1890.

9. Hayashi, K.; Hayashi, T.; Otsuka, H.; Takeda, Y. Antiviral activity of 5,6,7-trimethoxyflavone and its potentiation of the antiherpes activity of acyclovir. J. Antimicrob. Chemother. 1997, 39, 821-824, https://doi.org/10.1093/jac/39.6.82.

10. Sydiskis, R.J.; Owen, D.G.; Lohr, J.L.; Rosler, K.H.; Blomster, R.N. Inactivation of enveloped viruses by anthraquinones extracted from plants. Antimicrob. Agents Chemother. 1991, 35, 2463-2466, https://doi.org/10.1128/aac.35.12.2463.

11. Mosaddad, S.A.; Beigi, K.; Doroodizadeh, T.; Haghnegahdar, M.; Golfeshan, F.; Ranjbar, R.; Tebyanian, H. Therapeutic applications of herbal/synthetic/bio-drug in oral cancer: An update. Eur. J. Pharmacol. 2021, 890, 173657, https://doi.org/10.1016/j.ejphar.2020.173657.

12. Meruelo, D.; Lavie, G.; Lavie, D. Therapeutic agents with dramatic antiretroviral activity and little toxicity at effective doses: aromatic polycyclic diones hypericin and pseudohypericin. Proc. Natl. Acad. Sci. U. S. A. 1988, 85, 5230-5234, https://doi.org/10.1073/pnas.85.14.5230.

13. Schinazi, R.F.; Chu, C.K.; Ramesh Babu, J.; Oswald, B.J.; Saalmann, V.; Cannon, D.L.; Eriksson, B.F.H.; Nasr, M. Anthraquinones as a new class of antiviral agents against human immunodeficiency virus. Antiviral Res. 1990, 13, 265-272, https://doi.org/10.1016/0166-3542(90)90071-E.

14. Itai, A.; Mizutani, M.Y.; Tomioka, N. Computer-Assisted New Leocl Design. Guidebook on molecular modeling in drug design 1996, 93. 
15. Heidari, M.F.; Arab, S.S.; Noroozi-Aghideh, A.; Tebyanian, H.; Latifi, A.M. Evaluation of the substitutions in 212, 342 and 215 amino acid positions in binding site of organophosphorus acid anhydrolase using the molecular docking and laboratory analysis. Bratisl. Lek. Listy 2019, 120, 139, https://doi.org/10.4149/bll_2019_022.

16. Wadood, A.; Ahmed, N.; Shah, L.; Ahmad, A.; Hassan, H.; Shams, S. In-silico drug design: An approach which revolutionarised the drug discovery process. OA drug design \& delivery 2013, 1, 3.

17. Sharma, A.D.; Kaur, I. Eucalyptol (1,8 cineole) from Eucalyptus Essential Oil a Potential Inhibitor of COVID 19 Corona Virus Infection by Molecular Docking Studies; 2020; https://doi.org/10.20944/preprints202003.0455.v1.

18. Serseg, T.; Benarous, K.; Yousfi, M. Hispidin and Lepidine E: two Natural Compounds and Folic acid as Potential Inhibitors of 2019-novel coronavirus Main Protease (2019-nCoVMpro), molecular docking and SAR study. arXiv preprint arXiv:2004.08920 2020.

19. Khaerunnisa, S.; Kurniawan, H.; Awaluddin, R.; Suhartati, S.; Soetjipto, S. Potential Inhibitor of COVID-19 Main Protease (Mpro) From Several Medicinal Plant Compounds by Molecular Docking Study. Preprints.org: 2020; https://doi.org/10.20944/preprints202003.0226.v1.

20. Elfiky, A.A. Ribavirin, Remdesivir, Sofosbuvir, Galidesivir, and Tenofovir against SARS-CoV-2 RNA dependent RNA polymerase (RdRp): A molecular docking study. Life Sci. 2020, 253, 117592, https://doi.org/10.1016/j.1fs.2020.117592.

21. Aanouz, I.; Belhassan, A.; El-Khatabi, K.; Lakhlifi, T.; El-ldrissi, M.; Bouachrine, M. Moroccan Medicinal plants as inhibitors against SARS-CoV-2 main protease: Computational investigations. J. Biomol. Struct. Dyn. 2020, 1-9, https://doi.org/10.1080/07391102.2020.1758790.

22. Jeon, S.; Ko, M.; Lee, J.; Choi, I.; Byun, S.Y.; Park, S.; Shum, D.; Kim, S. Identification of Antiviral Drug Candidates against SARS-CoV-2 from FDA-Approved Drugs. Antimicrob. Agents Chemother. 2020, 64, e00819-00820, https://doi.org/10.1128/AAC.00819-20.

23. Colombier, M.-A.; Molina, J.-M. Doravirine: a review. Curr. Opin. HIV AIDS 2018, 13, 308-314, https://doi.org/10.1097/coh.0000000000000471.

24. Hardcastle, G.A.; Johnson, D.A.; Panetta, C.A.; Scott, A.I.; Sutherland, S.A. The Preparation and Structure of Hetacillin. The Journal of Organic Chemistry 1966, 31, 897-899, https://doi.org/10.1021/jo01341a060.

25. Sardana, V.; Burzynski, J.; Zalzal, P. Safety and efficacy of topical ketoprofen in transfersome gel in knee osteoarthritis: a systematic review. Musculoskeletal Care 2017, 15, 114-121.

26. Nosten, F.; Van Vugt, M.; Price, R.; Luxemburger, C.; Thway, K.L.; Brockman, A.; McGready, R.; Ter Kuile, F.; Looareesuwan, S.; White, N.J. Effects of artesunate-mefloquine combination on incidence of Plasmodium falciparum malaria and mefloquine resistance in western Thailand: a prospective study. The Lancet 2000, 356, 297-302.

27. Unni, S.; Aouti, S.; Thiyagarajan, S.; Padmanabhan, B. Identification of a repurposed drug as an inhibitor of Spike protein of human coronavirus SARS-CoV-2 by computational methods. J. Biosci. (Bangalore) 2020, 45, 130, https://doi.org/10.1007/s12038-020-00102-w. 